\begin{tabular}{|c|c|}
\hline Title & CMOS Smart Sensor for Monitoring the Quality of Perishables \\
\hline Author(s) & Ueno, K.; Hirose, T.; A sai, T .; A memiya, Y. \\
\hline Citation & $\begin{array}{l}\text { IEEE Journal of Solid-State Circuits, 42(4), 798-803 } \\
\text { https://doi.org/10.1109/JSSC.2007.891676 }\end{array}$ \\
\hline Issue Date & $2007-04$ \\
\hline Doc URL & http:/hdl.handle.net/2115/22218 \\
\hline Rights & $\begin{array}{l}\text { (c) } 2007 \text { IEEE. Personal use of this material is permitted. However, permission to reprint/republish this material for } \\
\text { advertising or promotional purposes or for creating new collective works for resale or redistribution to servers or lists, } \\
\text { or to reuse any copyrighted component of this work in other works must be obtained from the IEEE. IEEE, Journal of } \\
\text { Solid-State Circuits, V ol. 42, issue 4, 2007, pp. } 798-803\end{array}$ \\
\hline Tyре & article \\
\hline File Information & IJSC 42-4.pdf \\
\hline
\end{tabular}

Instructions for use 


\title{
CMOS Smart Sensor for Monitoring the Quality of Perishables
}

\author{
Ken Ueno, Student Member, IEEE, Tetsuya Hirose, Member, IEEE, Tetsuya Asai, Member, IEEE, and \\ Yoshihito Amemiya
}

\begin{abstract}
We developed a CMOS integrated-circuit sensor to monitor the change in quality of perishables that depends on surrounding temperatures. Our sensor makes use of the fact that the temperature dependence of the subthreshold current in MOSFETs is analogous to that of the degradation of perishables. The sensor is attached to perishable goods such as farm and marine products and is distributed from producers to consumers along with the goods. During their distribution process, the sensor measures the surrounding temperatures and emulates the degradation of the goods caused by the temperature. By reading the output of the sensor, consumers can determine whether the goods are fresh or not. Our sensor consists of subthreshold CMOS circuits with a low-power consumption of $10 \mu \mathrm{W}$ or lower.
\end{abstract}

Index Terms-CMOS, perishable, quality guarantee, smart sensor, subthreshold current, translinear.

\section{INTRODUCTION}

$\mathbf{C}$ ONTROLLING the quality of consumer goods in a distribution process is very important but not easy for perishables such as farm, marine, and dairy products. To guarantee the quality of these perishable goods, producers generally write an expiration date-for example, "Best before end: May '07" - on the package of the goods before shipping. However, producers have no way of knowing the conditions of distribution in detail— the goods might be distributed under good conditions, or they might be distributed under bad conditions with high temperatures and high humidity. To prepare for the worst, producers are obliged to set the expiration date of such products rather short. In consequence, considerable amounts of perishables are thrown away only because the expiration date has passed, though the goods are still edible. To solve this problem, Hirose et al. [1] proposed using a watchdog sensor that monitors the degradation of perishables during distribution from producers to consumers. This sensor is attached to the perishable goods and is distributed from producers to consumers along with the goods. It measures the surrounding temperatures and emulates the degradation of the goods caused by the temperature. By reading the sensor output, consumers can determine whether the goods are fresh or not. Hirose et al. [1] also proposed a method of emulation based on the analogy between the

Manuscript received August 25, 2006; revised December 3, 2006. This work is supported by VLSI Design and Education Center (VDEC), the University of Tokyo in collaboration with Cadence Design Systems, Inc.

The authors are with the Department of Electrical Engineering, Hokkaido University, Sapporo 060-0814, Japan (e-mail: k_ueno@sapiens-ei.eng. hokudai.ac.jp).

Digital Object Identifier 10.1109/JSSC.2007.891676 degradation rate of perishables and the subthreshold current of MOSFETs. They made a prototype chip consisting of MOSFET thermosensors and analog integrators consisting of operational amplifiers [2]. However, their sensor had difficulty in emulating chemical reactions with large activation energies because of its limited dynamic range: it was not able to emulate activation energy of more than $0.2 \mathrm{eV}$. Because the activation energy of spoiling reactions in most perishables is about $0.4-0.5 \mathrm{eV}$ [3], we must develop an improved sensor chip that can operate with such large values of activation energy.

Previously, we proposed an improved circuitry for watchdog sensors and confirmed through SPICE simulation that the sensor was able to emulate the degradation of perishables with a wide range of activation energy from 0.1 to $0.8 \mathrm{eV}$ [4]. This sensor circuit consists of MOSFET thermosensors, analog multipliers using a translinear circuit, and digital integrators. In this paper, we demonstrate the operation of our improved sensor with experimental results for sample chips made with a standard CMOS technology. Section II of this paper outlines the principle of the emulation for the degradation of perishables. Section III describes the processing architecture and the circuitry of the sensor we propose. Section IV shows the operation of the sensor with measurement results for various values of activation energy.

\section{EMUlation of THE DegRAdATION PROCESS}

\section{A. Expressing the Changes in Quality of Perishables}

The degradation process of perishables can be approximately expressed by a simple chemical reaction, $A+B \rightarrow C$, where $A$ and $B$ are the constituent elements of the perishables, and $C$ is an undesirable spoiling substance produced by the reaction [3]. The concentration of the spoiling substance $[C]$ can be given by

$$
[C]=[A]_{0}[B]_{0} k_{0} \int_{0}^{t_{1}} \exp \left(-\frac{E_{a}}{k_{B} T}\right) d t
$$

where $[A]_{0}$ and $[B]_{0}$ are the initial concentrations of constituents $A$ and $B$, and $E_{a}$ is the activation energy for the reaction. Concentration $[C]$ corresponds to the degradation and is greatly affected by the thermal history that the goods experience during their distribution process.

\section{B. Emulating the Degradation Process With CMOS Circuits}

To emulate the degradation process in (1) with electrical circuits, we make use of the transfer characteristics of a MOSFET operated in the subthreshold region as proposed by Hirose $e t$ $a l$. The explanation for (1)-(5) in the following is based on their papers [1] and [2]. The subthreshold current $I_{D}$ through 
a MOSFET is an increasing exponential function of the gatesource voltage $V_{G}$ and is given by

$$
I_{D}=I_{0} \exp \left(\frac{e\left(V_{G}-V_{T H}\right)}{\eta k_{B} T}\right)
$$

where $I_{0}$ is a process-dependent parameter, $e$ is the elementary charge, and $\eta$ is the subthreshold slope factor [5], [6]. To set an activation energy for emulating the degradation process, we prepared two MOSFETs (M1 and M2) biased by different gatesource voltages ( $V_{G 1}$ for M1 and $V_{G 2}$ for M2) and took the ratio of subthreshold currents ( $I_{D 1}$ for M1 and $I_{D 2}$ for M2) in the two MOSFETs. The ratio is given by

$$
\begin{aligned}
\frac{I_{D 2}}{I_{D 1}} & =\exp \left(-\frac{e\left(V_{G 1}-V_{G 2}\right)}{\eta k_{B} T}\right) \\
& =\exp \left(-\frac{E_{0}}{k_{B} T}\right)
\end{aligned}
$$

and

$$
E_{0}=\frac{e\left(V_{G 1}-V_{G 2}\right)}{\eta}
$$

where $E_{0}$ is the activation energy of the circuit-we call it the sensor activation energy. The variation of threshold-voltage $V_{T H}$ and process-dependent parameter $I_{0}$ are canceled by taking a ratio of two subthreshold currents. By integrating (3) with respect to time, we obtain

$$
\int_{0}^{t 1}\left(\frac{I_{D 2}}{I_{D 1}}\right) d t=\int_{0}^{t 1} \exp \left(-\frac{E_{0}}{k_{B} T}\right) d t .
$$

Because (5) is an electrical analog of (1), we can emulate the degradation of perishables by using subthreshold MOSFET circuits.

With this method, however, activation energy larger than $0.2 \mathrm{eV}$ cannot be implemented because, for large values of activation energy, the current ratio $I_{D 2} / I_{D 1}$ falls below $10^{-4}$ (e.g., $I_{D 2} / I_{D 1}=5 \times 10^{-5}$ for $0.25 \mathrm{eV}$ at room temperatures), and this makes it difficult to operate both MOSFETs in the subthreshold region, where the drain current is less than $1 \mu \mathrm{A}$. In other words, to set the activation energy to $0.25 \mathrm{eV}$ or more, current $I_{D 2}$ has to be set smaller than $10 \mathrm{pA}$ or less, but this is impractical because of leak currents in actual MOSFETs.

To emulate the degradation process of large activation energy, we constructed a sensor device consisting of a combination of analog and digital circuits, as shown in Fig. 1. The analog circuits emulate the degradation on the basis of (3) with a small value $E_{0}$ of activation energy, and the digital circuits process the output of the analog circuits in a manner shown later, thereby calculating (5) with an amplified activation energy $n E_{0}$ instead of $E_{0}$. With this construction, we can obtain large activation energy of $0.3 \mathrm{eV}$ or more, as shown in the following sections.

\section{Sensor Construction With Subthreshold CMOS CIRCUITS}

Fig. 2 shows a block diagram of the sensor we proposed. A thermosensor consists of two MOSFETs and produces two subthreshold currents $I_{D 1}$ and $I_{D 2}$, and the translinear divider calculates an output current $I_{\text {OUT }}$ proportional to the current

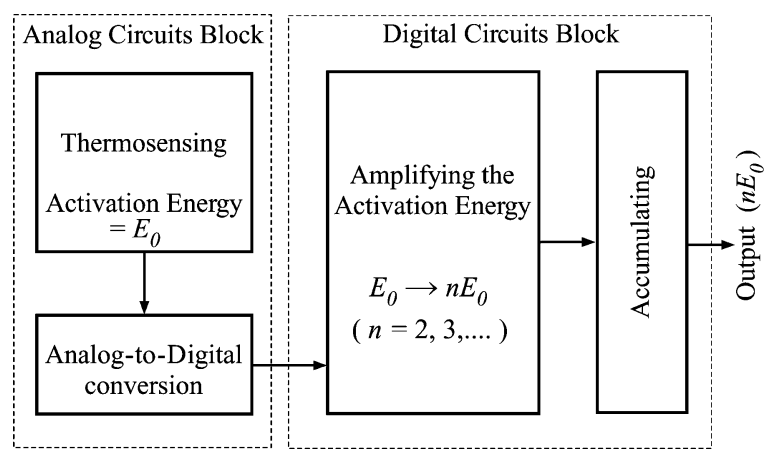

Fig. 1. Construction of our sensor device.

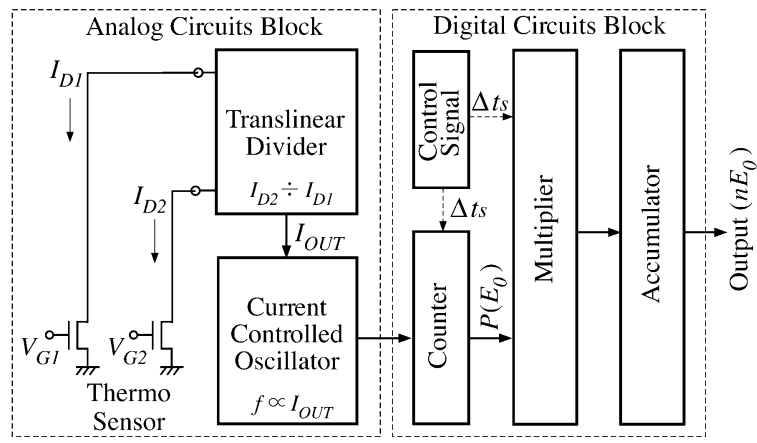

Fig. 2. Block diagram of the sensor device.

ratio $I_{D 2} / I_{D 1}$ given by (3), thereby emulating the rate equation for degradation. Sensor activation energy $E_{0}$ can be controlled by adjusting the difference between two bias voltages $V_{G 1}$ and $V_{G 2}$ for the thermosensor MOSFETs. The current-controlled oscillator (CCO) accepts current $I_{\mathrm{OUT}}$ and produces oscillation pulses with a frequency $f$ proportional to $I_{\text {OUT }}$. The digital counter records the number of the pulses at intervals of a short time $\Delta t_{s}$ to produce counting output $P\left(E_{0}\right)$, which is proportional to frequency $f$. To emulate the degradation process for large values of activation energy, the counting output is raised to a higher ( $n$ th) power by the digital multiplier and is stored in the accumulator. The output of the accumulator is an electrical analog of (1) with activation energy $n E_{0}$ instead of $E_{a}$-we call $n E_{0}$ the total activation energy. The details of each circuit are as follows.

\section{A. Thermosensor and Translinear Divider}

Fig. 3 shows the thermosensor and translinear divider used for emulating the rate equation for degradation. Given two input gate-source voltages $V_{G 1}$ and $V_{G 2}$, the thermosensor, or a pair of two MOSFETs (M1 and M2), produces the subthreshold currents $I_{D 1}$ and $I_{D 2}$ given by (2). The translinear divider accepts these currents through current mirrors and produces output current $I_{\mathrm{OUT}}$. In this circuit, the gate-source voltages ( $V_{1}$ through $V_{4}$ ) for the four MOSFETs (M3 through M6) form a closed loop, so we find that

$$
V_{1}-V_{2}+V_{3}-V_{4}=0 .
$$

From the translinear principle (see [7], [8] for details), we obtain

$$
I_{\mathrm{REF}} I_{D 2}=I_{D 1} I_{\mathrm{OUT}}
$$




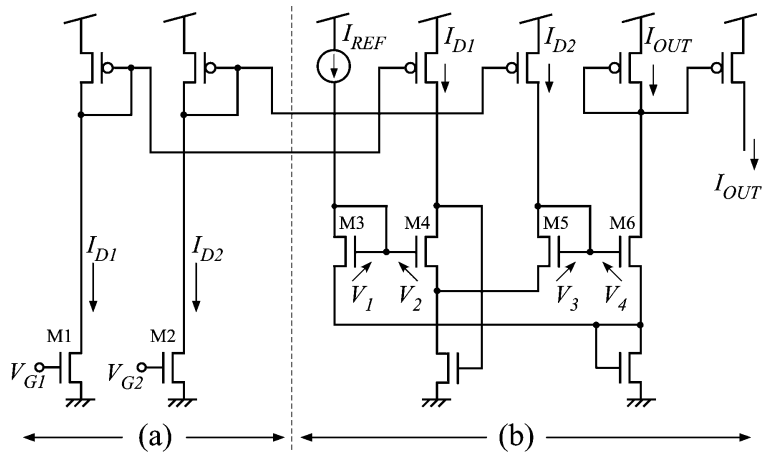

Fig. 3. Circuit configuration of (a) thermosensor and (b) translinear divider circuit, consisting of MOSFETs operated in the subthreshold region. $I_{\mathrm{REF}}$ is a constant current independent of temperature. The activation energy of the circuit can be controlled by adjusting $V_{G 1}-V_{G 2}$.

where $I_{\mathrm{REF}}$ is a temperature-independent reference current for the translinear divider. The output current is proportional to $I_{D 2} / I_{D 1}$ and given by

$$
\begin{aligned}
I_{\mathrm{OUT}} & =I_{\mathrm{REF}} \frac{I_{D 2}}{I_{D 1}} \\
& =I_{\mathrm{REF}} \exp \left(-\frac{E_{0}}{k_{B} T}\right)
\end{aligned}
$$

where

$$
E_{0}=\frac{e\left(V_{G 1}-V_{G 2}\right)}{\eta}
$$

Therefore, the output current can emulate the rate (1) for degradation. Sensor activation energy $E_{0}$ can be controlled by adjusting the difference between the two bias voltages $V_{G 1}$ and $V_{G 2}$.

\section{B. Current Controlled Oscillator (CCO)}

The current controlled oscillator (CCO) is used for producing the oscillation pulses proportional to the output current of the translinear divider $I_{\mathrm{OUT}}$. Fig. 4 shows the $\mathrm{CCO}$ consisting of inverters connected in a ring. The oscillation frequency $f$ depends on the applied current $I_{\mathrm{OUT}}$ and is given by

$$
\begin{aligned}
f & =\frac{I_{\mathrm{OUT}}}{2 m A C_{L} V_{\mathrm{dd}}} \\
& =\frac{I_{\mathrm{REF}}}{2 m A C_{L} V_{\mathrm{dd}}} \exp \left(-\frac{E_{0}}{k_{B} T}\right)
\end{aligned}
$$

where $m$ is the number of inverters in the oscillator, $C_{L}$ is the load capacitance for each inverter, and $A$ is the delay fitting parameter (about 0.75) [9]. Since the voltage of button batteries has little temperature dependence, oscillation frequency $f$ depends on only $\exp \left(-E_{0} / k_{B} T\right)$. Therefore, oscillation frequency $f$ is approximately proportional to current $I_{\text {OUT }}$ of the translinear divider.

\section{Digital Counter}

The digital counter consists of D-flip-flops and counts the number of CCO oscillation pulses at short time intervals of $\Delta t_{s}$.

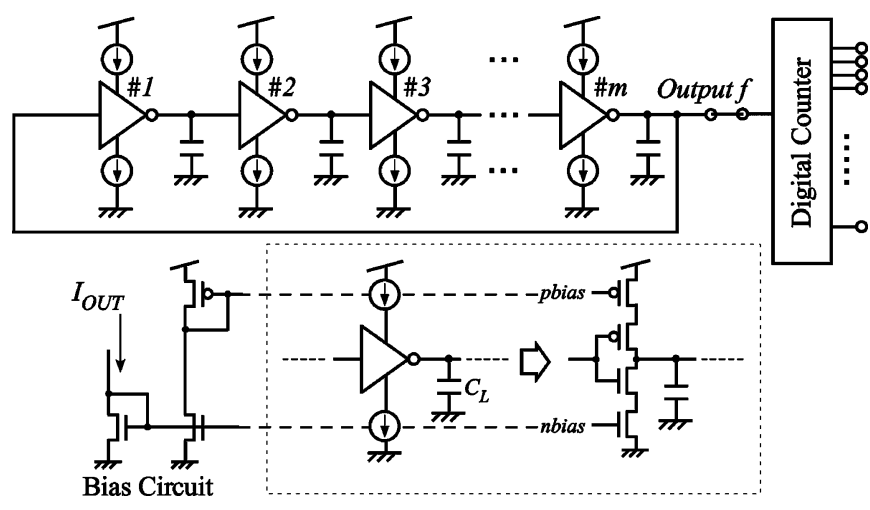

Fig. 4. Current controlled oscillator consisting of inverters connected in a ring. Oscillation frequency $f$ is proportional to input current $I_{\text {OUT }}$.

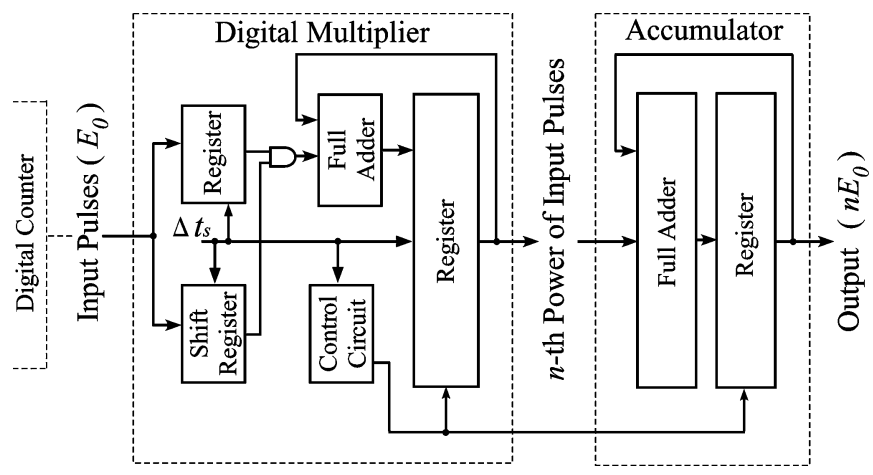

Fig. 5. Flow chart of the digital processing: serial multiplication and accumulation.

The counting output $P\left(E_{0}\right)$ at $\Delta t_{s}$ intervals is expressed by

$$
P\left(E_{0}\right)=\int_{t}^{t+\Delta t_{s}} f d t .
$$

From this, we can calculate the degree of degradation.

\section{Digital Multiplier and Accumulator}

To emulate the degradation for large activation energy, we raised the counting output to a higher ( $n$ th) power at every $\Delta t_{s}$ seconds by using a digital multiplier and stored the raised data in an accumulator. Fig. 5 shows the flow chart of the digital processing; the multiplication is performed in a serial manner with registers and adders. The output of the multiplier at $\Delta t_{s}$ intervals is given by

$$
P\left(E_{0}\right)^{n}=\left(\int_{t}^{t+\Delta t_{s}} f d t\right)^{n} .
$$

On condition that temperature (or frequency $f$ ) is almost constant during short time $\Delta t_{s}$ (this is true for $\Delta t_{s}$ of a few seconds or minutes), the equation can be rewritten as

$$
\begin{aligned}
P\left(E_{0}\right)^{n} & =\left(\int_{t}^{t+\Delta t_{s}} f^{n} d t\right) \\
& =P\left(n E_{0}\right) .
\end{aligned}
$$




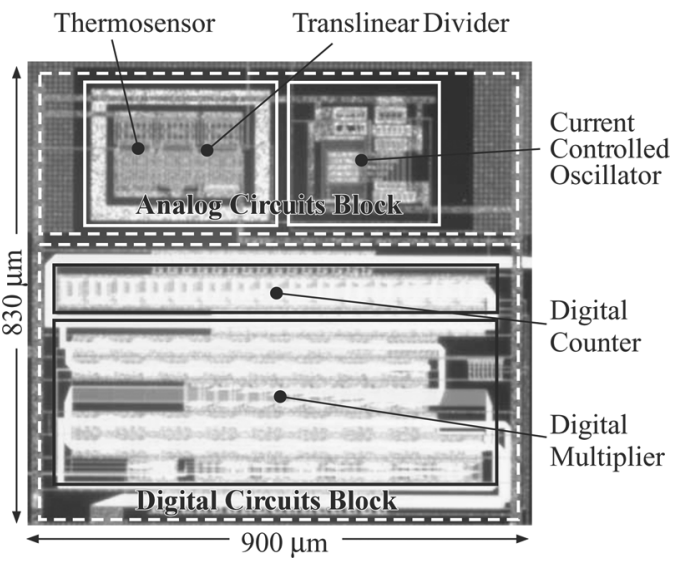

Fig. 6. Chip micrograph of the sensor, designed with $0.35-\mu \mathrm{m}, 2$-poly, 5-metal CMOS process parameters. The area is $900 \times 830 \mu \mathrm{m}$, excluding $\mathrm{I} / \mathrm{O}$ pads and connections.

This way, sensor activation energy $E_{0}$ can be increased to the total activation energy $n E_{0}$. The output of the accumulator at time $t_{1}$ is represented by

$$
\text { output }=\left(\frac{I_{\mathrm{REF}}}{2 m A C_{L} V_{\mathrm{dd}}}\right)^{n} \int_{0}^{t_{1}} \exp \left(-\frac{n E_{0}}{k_{B} T}\right) d t .
$$

This is analogous to (1); therefore we can emulate the process of degradation with large values of activation energy.

When the value of the accumulator exceeds a certain threshold value set up in advance, we can determine that the product has expired or not.

\section{RESULTS}

We made a prototype sensor with a $0.35-\mu \mathrm{m}, 2$-poly, 5-metal CMOS process and a $1.5-\mathrm{V}$ power supply (nominal voltage of dry manganese batteries). A CCO was implemented by fivestage current-starved inverters connected in a ring with load capacitance $C_{L}=0.5 \mathrm{pF}$. The raising factor of the digital multiplier was set to 2. Fig. 6 shows the chip micrograph. The chip area is $900 \mu \mathrm{m} \times 830 \mu \mathrm{m}$, excluding $\mathrm{I} / \mathrm{O}$ pads and connections.

Fig. 7 shows the drain-current versus gate-voltage curve of a thermosensor MOSFET for temperatures from $-20^{\circ} \mathrm{C}$ to $100^{\circ} \mathrm{C}$. The activation energy of the output $\left[I_{\mathrm{OUT}}\right.$ in $\left.(8)\right]$ of the translinear divider was controlled by adjusting the difference between the two bias voltages $V_{G 1}$ and $V_{G 2}$; we fixed $V_{G 1}$ to $470 \mathrm{mV}$ and changed $V_{G 2}$ from 190 to $400 \mathrm{mV}$, which corresponds to the sensor activation energy from 0.05 to $0.2 \mathrm{eV}$.

Fig. 8 shows the output current of the translinear divider with different values of activation energy. The vertical axis represents the output current at a log-scale, and the horizontal axis represents the reciprocal of temperature. The activation energy was set in a range of $0.05-0.2 \mathrm{eV}$. Reference current $I_{\mathrm{REF}}$ was set to $500 \mathrm{nA}$. The points in the figure show the measurement results, and the dashed lines show the theoretical values obtained from (8). The logarithm of the output current is proportional to the reciprocal of temperature, and this is consistent with (8).

Fig. 9 shows the output waveforms of the $\mathrm{CCO}$ measured at different temperatures from $-20^{\circ} \mathrm{C}$ to $100^{\circ} \mathrm{C}$, where the sensor activation energy $\left(E_{0}=\left(V_{G 1}-V_{G 2}\right) / \eta\right)$ was set to $0.2 \mathrm{eV}$. The

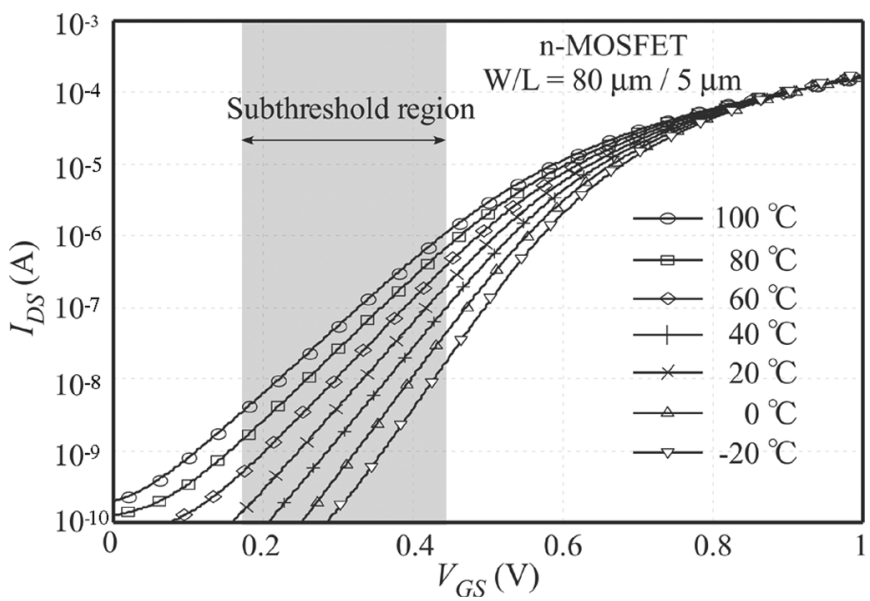

Fig. 7. Drain current $I_{D S}$ of thermosensor MOSFETs (n-channel, $W / L=$ $80 \mu \mathrm{m} / 5 \mu \mathrm{m})$ as a function of gate-source voltage $V_{G S}-I_{D S}$.

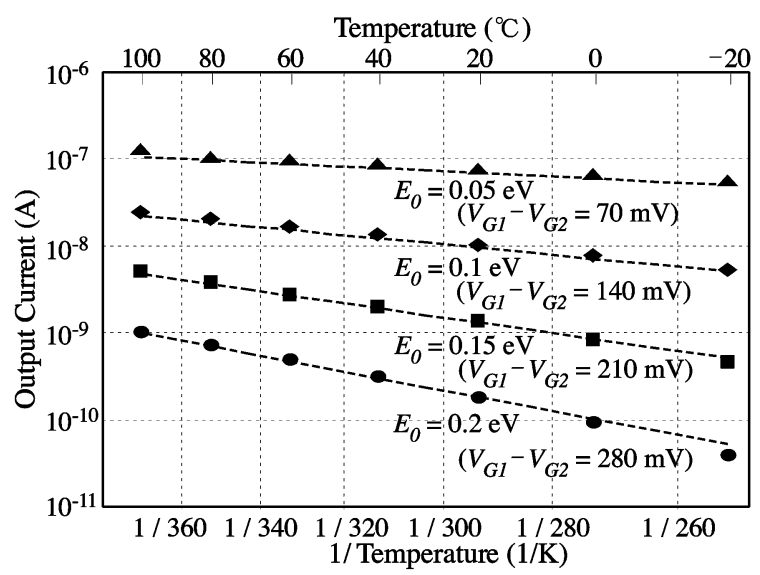

Fig. 8. Output current $I_{\mathrm{OUT}}$ of the translinear divider measured at temperatures from $-20^{\circ} \mathrm{C}$ to $100^{\circ} \mathrm{C}$, with sensor activation energy $E_{0}$ as a parameter

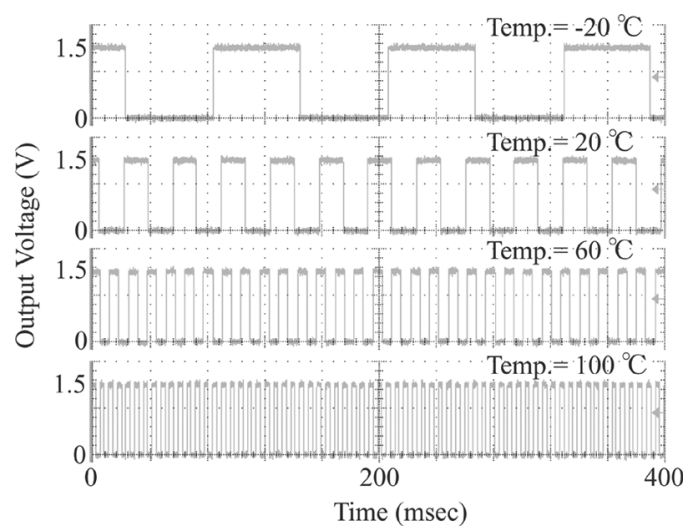

Fig. 9. CCO output waveforms measured at different temperatures. The sensor activation energy $E_{0}$ was set at $0.2 \mathrm{eV}$.

frequency (10) of the $\mathrm{CCO}$ oscillation increased with increasing temperature from $8 \mathrm{~Hz}$ to $160 \mathrm{~Hz}$. The counter accepted the oscillation output and sent the counted data to the multiplier at intervals of short time $\Delta t_{s}$ (= a few seconds). Fig. 10 shows the frequency of oscillation measured as a function of the reciprocal of temperature, with $V_{G 1}-V_{G 2}$ as a parameter. The value of sensor activation energy $E_{0}$ was $0.05 \mathrm{eV}$ for $V_{G 1}-V_{G 2}=$ 


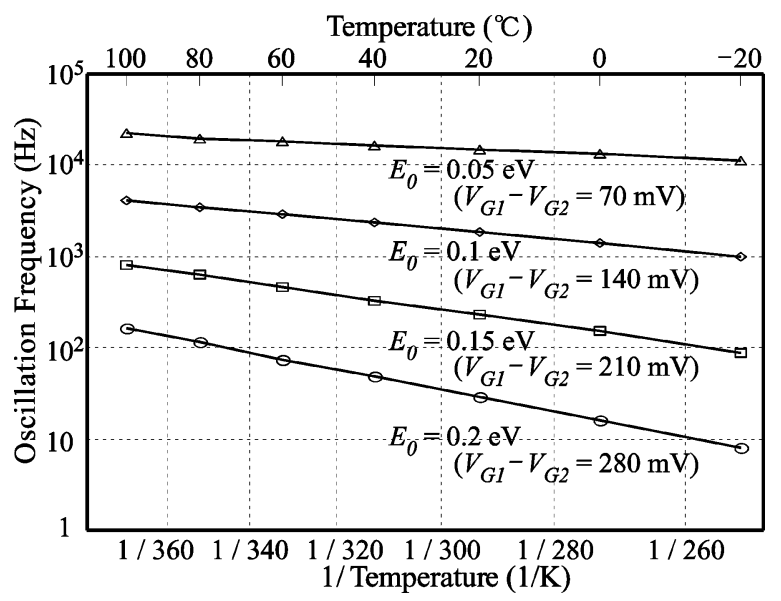

Fig. 10. Oscillation frequency of the $\mathrm{CCO}$ measured at temperatures from $-20^{\circ} \mathrm{C}$ to $100^{\circ} \mathrm{C}$, with sensor activation energy $E_{0}$ as a parameter.

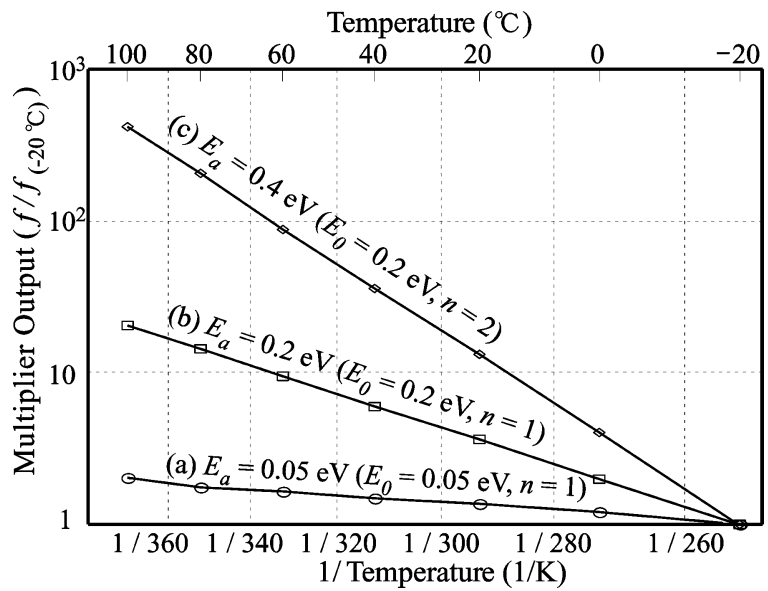

Fig. 11. Multiplier output measured as a function of temperature for sensor activation energies $E_{0}=0.05 \mathrm{eV}$ and $0.2 \mathrm{eV}$. Plotted for raising factors $n=1$ [lines (a) and (b)] and $n=2$ [line (c)], and normalized to the value at $-20^{\circ} \mathrm{C}$. A large value, $0.4 \mathrm{eV}$, of total activation energy $E_{a}$ can be achieved by secondpower raising.

$70 \mathrm{mV}$ and $0.2 \mathrm{eV}$ for $V_{G 1}-V_{G 2}=280 \mathrm{mV}$. As (10) shows, the oscillation frequency is an exponential function of the reciprocal of temperature.

Fig. 11 shows the output (13) of the multiplier (i.e., the input for the accumulator) as a function of temperature. Because multiplier output which emulates large activation energy has large temperature dependence, the output was normalized at the value of $-20^{\circ} \mathrm{C}$. The results are for sensor activation energy $E_{0}$ and raising factor $n$ of $E_{0}=0.05 \mathrm{eV}$ and $n=1$ for (a), $E_{0}=0.2 \mathrm{eV}$ and $n=1$ for (b), and $E_{0}=0.2 \mathrm{eV}$ and $n=2$ for (c), where each output is normalized to the value at $-20^{\circ} \mathrm{C}$. Large values of total activation energy $E_{a}$ was thus able to be achieved by adjusting bias voltage $V_{G 1}-V_{G 2}$ and raising factor $n$.

To confirm the total operation of our sensor, we set the sensor chip at various temperatures and emulated the degradation of a perishable with a given activation energy. Fig. 12 shows the results. In this example, we set the chip in a thermostatic chamber and changed its temperature with time in a range from $-20^{\circ} \mathrm{C}$ to $100^{\circ} \mathrm{C}$. The dashed curve is the result for a total activation energy of $0.2 \mathrm{eV}\left(E_{0}=0.2 \mathrm{eV}, n=1\right)$, and the solid curve

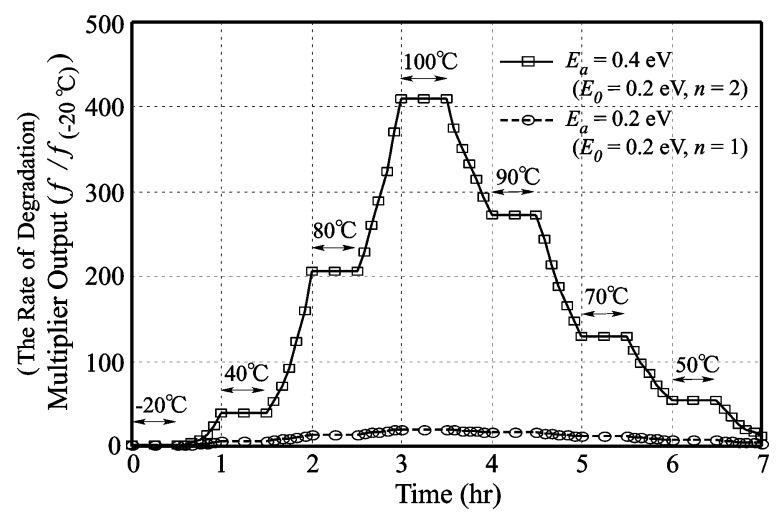

Fig. 12. Multiplier output as a function of time, measured at changing temperatures for a total activation energy of $0.2 \mathrm{eV}\left(E_{0}=0.2 \mathrm{eV}, n=1\right)$ and $0.4 \mathrm{eV}$ $\left(E_{0}=0.2 \mathrm{eV}, n=2\right)$. Each output is normalized to the value at $-20^{\circ} \mathrm{C}$. The integral of the output curve corresponds to the output of the accumulator, i.e., the amount of the degradation.

TABLE I

PERFORMANCE SUMMARY

\begin{tabular}{c|c}
\hline Process & $0.35-\mu \mathrm{m}, 2$-poly, 5-metal CMOS \\
Temperature range & $-20-100{ }^{\circ} \mathrm{C}$ \\
$V_{D D}$ & $1.5 \mathrm{~V}$ \\
Activation energy & adjustable from $0.05-0.4 \mathrm{eV}$ \\
Power & $9.7 \mu \mathrm{W}\left(T=100{ }^{\circ} \mathrm{C}\right)$ \\
Chip Area & $900 \times 830 \mu \mathrm{m}$ \\
Multiplier & $8 \mathrm{bit}$ \\
Raising factor $(n)$ & 2 \\
Error of emulated activation energy & $\pm 6 \%$ \\
\hline
\end{tabular}

is for $0.4 \mathrm{eV}$ ( $E_{0}=0.2 \mathrm{eV}, n=2$ ), where each output is normalized to the value at $-20{ }^{\circ} \mathrm{C}$. The vertical axis represents the multiplier output (13), or the rate of degradation, and the integral of each curve represents the accumulator output (14), or the amount of degradation. It is shown that the degradation proceeded rapidly at high temperatures. From the accumulator output, we can know the amount of degradation and can determine whether perishables are fresh or not. By adjusting the bias voltage $V_{G 1}-V_{G 2}$ and raising factor $n$, a wide range of activation energy can be achieved.

Table I summarizes the performance of our sensor chip. The chip showed the maximum power consumption, $9.7 \mu \mathrm{W}$, at $0.05-\mathrm{eV}$ activation energy and $100^{\circ} \mathrm{C}$. This means that our sensor can work for years with a small-sized button battery. The error of emulated activation energy was $\pm 6 \%$. In our prototype chip, the raising factor of the digital multiplier was set to 2 . If we have to emulate larger activation energy more than $0.4 \mathrm{eV}$, the digital multiplier which can perform higher power computation should be implemented.

\section{CONCLUSION}

We developed a CMOS watchdog sensor to monitor the quality of perishables in their distribution process. This sensor performs monitoring by making use of the analogy between the temperature dependence of degradation and that of subthreshold MOSFET current. We fabricated a prototype chip with a $0.35-\mu \mathrm{m}$ CMOS process and demonstrated its operation. 
A wide range of activation energy can be achieved by using a combination of analog and digital circuits. The power consumption of the sensor is only $10 \mu \mathrm{W}$ at $100^{\circ} \mathrm{C}$, so the sensor can work for years even with a small-sized button battery. The wide controllability of activation energy enables us to emulate the degradation of most perishables such as farm, marine, dairy products, sake and wine, and medical supplies.

\section{REFERENCES}

[1] T. Hirose, R. Yoshimura, T. Ido, T. Matsuoka, and K. Taniguchi, "Watchdog circuit for product degradation monitor using subthreshold MOS current," in Proc. Int. Conf. Solid State Devices and Materials, Sep. 2004, pp. 150-151.

[2] T. Hirose, R. Yoshimura, T. Ido, T. Matsuoka, and K. Taniguchi, "Watch-dog circuit for quality guarantee with subthreshold MOSFET current," IEICE Trans. Electron., vol. E87-C, no. 11, pp. 1910-1914, Nov. 2004

[3] P. W. Atkins, Physical Chemistry, 6th ed. Oxford, UK: Oxford Univ. Press, 1998

[4] K. Ueno, T. Hirose, T. Asai, and Y. Amemiya, "A CMOS watch-dog sensor for guaranteeing the quality of perishables," in Proc. Int. Conf. Sensors, Oct. 2005, pp. 1209-1212.

[5] Y. Taur and T. H. Ning, Fundamentals of Modern VLSI Devices. Cambridge, UK: Cambridge University Press, 2002.

[6] P. R. Gray and R. G. Meyer, Analysis and Design of Analog Integrated Circuits, 3rd ed. New York: Wiley, 1993.

[7] S.-C. Liu, J. Kramer, G. Indiveri, T. Delbruck, and R. Douglas, Analog VLSI: Circuits and Principles. Cambridge, MA: MIT Press, 2002.

[8] E. S. Sinencio and A. G. Andreou, Low-Voltage/Low-Power Integrated Circuits and Systems. New York: IEEE, 1999.

[9] B. H. Calhoun, A. Wang, and A. Chandrakasan, "Modeling and sizing for minimum energy operation in subthreshold circuits," IEEE J. SolidState Circuits, vol. 40, no. 9, pp. 1778-1786, Sep. 2005.

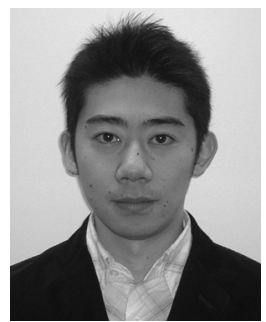

Ken Ueno ( $\left.S^{\prime} 05\right)$ was born in Hokkaido, Japan, in 1979. He received the B.S. degree in electronics and information engineering from Hokkai-Gakuen University, Sapporo, Japan, in 2002. He is currently working toward the M.E. degree in electrical engineering at Hokkaido University, Sapporo, Japan.

His current research interests are in low-power analog CMOS circuits.

Mr. Ueno is a member of the Institute of Electronics, Information and Communication Engineers of Japan, and the IEEE.

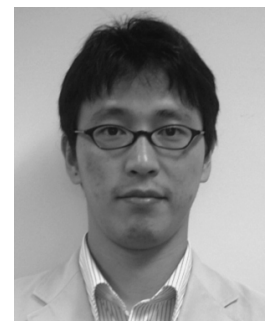

Tetsuya Hirose (M'05) received the B.S., M.S., and $\mathrm{Ph}$.D. degrees from Osaka University, Osaka, Japan, in 2000, 2002, and 2005, respectively.

Currently, he is a Research Associate in the Department of Electrical Engineering, Hokkaido University, Sapporo, Japan. His current research interests are in low-power analog/digital CMOS circuits and subthreshold MOSFET functional circuits for intelligent sensors.

Dr. Hirose is a member of the Institute of Elecof Japan, and the IEEE. tronics, Information and Communication Engineers

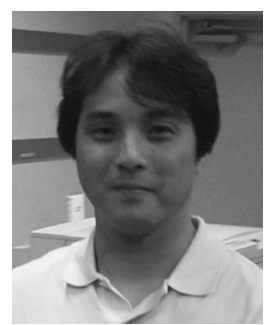

Tetsuya Asai (M'01) received the B.S. and M.S. degrees in electrical engineering from Tokai University, Kanagawa, Japan, in 1993 and 1996, respectively, and the Ph.D. degree in electrical and electronic engineering from Toyohashi University of Technology, Aichi, Japan, in 1999.

$\mathrm{He}$ is now an Associate Professor in the Department of Electrical Engineering, Hokkaido University, Sapporo, Japan. His current research interests include nonlinear analog processing in neural networks and reaction-diffusion systems as well as design and applications of neuromorphic VLSIs.

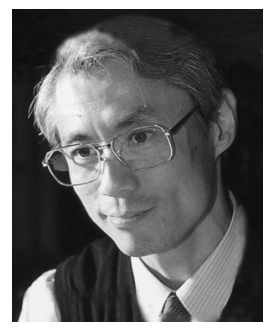

Yoshihito Amemiya received the B.E., M.E., and $\mathrm{Ph} . \mathrm{D}$. degrees from the Tokyo Institute of Technology, Tokyo, Japan, in 1970, 1972, and 1975, respectively.

He joined NTT Musashino Laboratories in 1975, where he worked on the development of silicon process technologies for high-speed logic LSIs. From 1983 to 1993, he was with NTT Atsugi Laboratories and developed bipolar and CMOS circuits for Boolean logic LSIs, neural network LSIs, and cellular automaton LSIs. Since 1993, he has been a Professor with the Department of Electrical Engineering, Hokkaido University, Sapporo, Japan. His research interests are in the fields of silicon LSI circuits, signal processing devices based on nonlinear analog computation, logic systems consisting of single-electron circuits, and information processing devices making use of quantum nanostructures. 